\title{
Nurse-Led Interventions in the De-Addiction Setting: Current State of Evidence
}

\author{
Jaison Joseph $^{1 *}$, Dr.Deepika C Khakha ${ }^{2}$, Biji P Varkey ${ }^{3}$ \\ ${ }^{1}$ Lecturer (Jr), College of Nursing, Pt. B.D. Sharma University of health Sciences, Rohtak India \\ ${ }^{2}$ Associate professor, College of Nursing, All India Institute of medical Sciences, New Delhi, India \\ ${ }^{3}$ Nursing Officer, Post Graduate Institute of Medical Sciences, Rohtak
}

*Corresponding Author: Jaison Joseph, Lecturer (Jr), College of Nursing, Pt. B.D. Sharma University of health Sciences, Rohtak India.Ph:9468277298, Email: jaisonjsph@yahoo.com

\begin{abstract}
Substance abuse is one of the most life-threatening illnesses worldwide, which can be associated with adverse consequences related to repeated use of the substance. Nurses are directly involved in providing care for patients with substance abuse disorder. Therefore, they are in a key position to help patients suffering from substance abuse. However, the nurse's role in the assessment and management of substance abuse is not well-defined. There is substantial data available on various nurse-led interventions on reducing substance use disorders and are majorly based on preventive and psychosocial interventions. The present article provides an account of the summary of the involvement of nurses in the various deaddiction setting. Nurses should acknowledge the extent of the problem and the importance of various interventions for alcohol and other psychoactive substances. There is a need to develop standards and implementation protocols as per the area of involvement such as PHCs, general hospital wards, or outpatient settings. Screening must be incorporated with routine clinical practice when assessing new patients and during periodic reviews. As the largest members of the health care team, nurses can make a significant impact on reducing substance use disorders by engaging in related interventions. But nurses lack courage due to time inadequacy, lack of confidence, and knowledge. Education, training \& evidence-based researches can only help the nurses for the effective implementation of these interventions.
\end{abstract}

Keywords: Nurse-led interventions, de-addiction

\section{INTRODUCTION}

Substance abuse is one of the most lifethreatening illnesses worldwide, which can be associated with adverse consequences related to repeated use of the substance. Untreated substance abuse might result in disturbances in social, occupational, or recreational activities. Furthermore, intoxication and withdrawal might have devastating impacts on health. [1] Substance use disorder (SUD) is a prevalent health issue with serious personal and societal consequences. SUDs are often associated with various physical health problems [2, 3], comorbid with other psychiatric disorders [4] and implicated in significant social and economic consequences. [5, 6] The substantial increases in access to care for SUDs have led to a greater emphasis on evaluating interventions to identify best practices for SUD treatment in healthcare systems. [7] There is no single intervention appropriate for all individuals with substance abuse. The effective intervention should consider the multiple needs of the individual with substance abuse, not only drug use.

\section{Pathways of CaRe in De-AdDiction: ROLE OF NURSE}

Nurses are directly involved in providing care for patients with substance abuse disorder. Therefore, they are in a key position to help patients suffering from substance abuse. However, the nurse's role in the assessment and management of substance abuse is not welldefined. [8 Nurses should anticipate addiction and be aware of their risk factors. They should accurately assess patients and immediately report any signs and symptoms of addiction. Sudden change in behaviors and personality, social isolation, poor occupational performance, preoccupation with substances abused, and sudden weight loss are among the commonly reported symptoms of substance abuse. Nurses need to know the most common substances that cause abuse such as Alcohol, Amphetamines, Caffeine, Cannabis, Cocaine, Hallucinogens, 
Inhalants, Nicotine, Opioids, Phencyclidine, Sedative, and hypnotics, or anti-anxiety agents. To better assess substance abuse in patients, nurses should use specific and valid screening tools such as the CAGE questionnaire for screening of Alcoholism. Furthermore, nurses should be aware of the laboratory tests used to assess patients with substance abuse such as blood alcohol levels. During the assessment phase, nurses should examine their feelings and avoid stigmatizing patients with substance abuse. [9]

Nurses should help patients to overcome many barriers that negatively influence attitudes toward seeking professional help, including lack of knowledge, denial, comorbidity, embarrassment, and lack of support. Nurses can participate in different management modalities for patients with substance abuse disorders such as conducting individual therapy for clients with the substance-related disorder who have high levels of anxiety and inadequate coping mechanisms. [10]

Nurses can discuss with the patient possibility of relapse and refusal to continue therapy. In a group setting, nurses can facilitate group discussions to enhance sobriety and willingness to share experiences. The adequate period spent in treatment is critical to achieving sobriety. Nurses can also instruct patients to do behavioral modifications and use specific medications such as Disulfiram (Antabuse). No specific antidotes are available for all substances abused, and each substance usually has a specific antidote to be used. During the detoxification process, the nurse should provide a calm and supportive environment for the patient and administer the needed therapy as ordered. Most symptoms of intoxication are self-limited and resolve within a few days after the supportive treatment. During the intoxication process, most patients experience acute symptoms such as anxiety, agitation, and tremor. Most of the time, anxiety, agitation, and seizures are treated by benzodiazepines.

For the severe agitation, antipsychotics are used. [11] Abrupt discontinuation of the substances abused is usually not recommended. However, some substances such as stimulants and hallucinogens can be discontinued without tapering off or replacement with another therapy as they have rare and low-grade withdrawal symptoms. [12] If necessary, intravenous fluids and antiemetics are administered during supportive care. Unsurprisingly, patients with severe intoxication symptoms should have frequent neurological assessments and be initially attached to continuous cardiac monitoring with pulse oximetry. [13]

When the condition of the patient becomes better, nurses should promote understanding of the physical symptoms and how to deal with them. The nurse can explain to the patient the causes of substance abuse. Immediately before discharge, the nurse should encourage continued participation in outpatient support systems and long-term treatment. Patients should be also instructed about alternative sources of satisfaction and coping strategies. The patient should acknowledge the relationship between everyday life problems and substance use. Nurses should tell patients that substance abuse disorder is a mental illness like schizophrenia and depression and could be associated with poor coping strategies. [14] Interventions directed toward increased self-worth are encouraged. Safety is an important aspect that should be prioritized to assure that the patient has not caused harm to self or others or caused any physical injury to others. The most important point before discharge is to support the patient to assume responsibility for his or her behavior and to verbalize the importance of abstaining from the use of substances. Finally, after discharge, a longterm follow-up is recommended, and the nurse can work as a liaison between the patient and the community services. [15]

There is substantial data available on various nurse-led interventions on reducing substance use disorders and are majorly based on preventive and psychosocial interventions. Various approaches in preventive interventions include school-based prevention programmes [16], family-based prevention programmes [17], restricting or banning on related advertisements. [18] Most of the nurse-led interventions are psychosocial interventions that aim to cut down or reduce current pattern substance use and incorporates strategies such as motivational interviewing, cognitive-behavioral therapy [19] and screening, and brief interventions. Brief intervention is a time-limited psychological treatment strategy in which structured therapy of short duration is offered to assist an individual to stop or reduce harmful or hazardous drinking. Screening is often offered before the brief intervention and the length of the intervention is determined by the risk levels of alcohol use as per the screening instrument. [20]

\subsection{Nurse-Led Screening and Brief Interventions in the De-Addiction Setting}

As the biggest component of the health care workforce, nurses can make a significant impact 
in reducing the harmful use of alcohol by engaging in screening and BIs. Empirical evidence exists for the involvement of nurses in the delivery of BIs for hazardous or harmful use of alcohol in primary health care and inpatient hospital settings. [21, 25] A systematic review compared the efficacy of nurse-conducted brief interventions (NCBIs) in reducing alcohol consumption, by looking at with treatment as usual compared to other treatments and general physician delivered brief interventions. The review concluded that five trials reported a statistically significant reduction in alcohol consumption in the intervention group with 6-12 months to follow up period in which two trials having the opinion that BI delivered by nurses was as efficacious as by physicians. Empirical evidence exists for the involvement of nurses and midwives in the delivery of brief interventions for hazardous or harmful use of alcohol in primary health care and inpatient hospital settings. [26, 36]

The evidence for nursing involvement in screening and brief interventions in hospital emergency departments is weaker, and it may be that emergency departments, where patients often present with acute or critical health needs, are not suitable environments for discussion of a topic such as substance use.

Findings from studies of brief interventions conducted in outpatient clinics, during an appointment after initial treatment of an injury, indicate that this is an area where screening and brief interventions can be very effective. The studies that tested interventions delivered to hospital inpatients used relatively small samples, but the results suggest that the interventions had been effective. [37, 44]

Although nurses working in primary health care generally assess patients' alcohol use, it appears that they seldom use validated screening tools. While nurses do give advice and information to patients whom they consider to be hazardous and harmful drinkers, there is some evidence that they are working from a low knowledge base. As a consequence, the interventions may be inappropriate, or opportunities to intervene may be missed. Findings from this review suggest that few hospital nurses routinely screen patients for alcohol use, and there is little evidence of nurses' or midwives' practice regarding screening and brief interventions for the hazardous and harmful use of other substances. Many studies pointed out thepractice of nurse-led interventions in the de-addiction setting. [45, 54]

\subsection{Nurse-Led Cognitive -Behavioral Interventions in De-Addiction Settings}

The various components of cognitive-behavioral interventions were used for decreasing substance abuse among the various population. The available literature suggests the involvement of nurses as a therapist in reducing substance use in diverse settings such as school health setting. [55] The nurse's role in delivering family-based therapies includes counseling, promotion of self-care activities, developing strengths and resources, providing supportive therapy, education, health teaching, and ultimately, building resiliency in youths and their families. [56] Nurses also address adolescents' substance use and related problems by enhancing family function through building skills related to communication and conflict resolution. [57] Interventions may include developing contracts to reinforce behaviors associated with abstinence from drugs, implementing skill-based interventions and training, developing communication skills, and facilitating access to education and training opportunities that can help the young person develop the skills necessary to obtain employment or attend school. [58]

\subsection{Nurse-Led Interventions on Decreasing Substances among Homeless Youth}

A community-based participatory action pilot study assessed the impact of a nurse-led intervention on decreasing the use of drugs and alcohol among a sample of homeless young adults $(\mathrm{N}=154)$ visiting a drop-in site in Santa Monica, California. The program consisted of an HIV/AIDS and Hepatitis Health Promotion (HHP) program led by nurses. Six-month follow-up data were obtained from 100 of these individuals. Findings revealed significant reductions in alcohol and marijuana use and binge drinking. The successful outcomes of the study intervention recommend the utility of nurse-led promotion strategies to decrease drug and alcohol use and other risky behaviors in homeless youth populations. [55]

\subsection{Nurse-Led Interventions in De-Addiction Settings Future Directions}

Nurses should acknowledge the extent of the problem and the importance of various interventions for alcohol and other psychoactive substances. There is a need to develop standards and implementation protocols as per the area of involvement such as PHCs, general hospital wards, or outpatient settings. Screening must be 
incorporated with routine clinical practice when assessing new patients and during periodic reviews. Moreover, continuous education is required to utilize the available resources such as validated screening tools and manuals for the delivery of various interventions, amended as necessary for a range of clinical settings;

\section{CONCLUSION}

As the largest members of the health care team, nurses can make a significant impact on reducing substance use disorders by engaging in related interventions. But nurses lack courage due to time inadequacy, lack of confidence, and knowledge. Education, training \& evidencebased researches can only help the nurses for the effective implementation of these interventions.

\section{REFERENCES}

[1] Degenhardt L, Hall W. Extent of illicit drug use and dependence, and their contribution to the global burden of disease. Lancet 2012; 379:5570.

[2] Cargiulo T. Understanding the health impact of alcohol dependence. Am J Health Syst Pharm 2007; 64:S5-S11.

[3] Rehm J. The risks associated with alcohol use and alcoholism. Alcohol Res Health 2011; 34:135-143.

[4] Grant BF, Goldstein RB, Saha TD, et al. Epidemiology of DSM-5 alcohol use disorder: results from the National Epidemiologic Survey on Alcohol and Related Conditions III. JAMA Psychiatry 2015; 72:757-766.

[5] World Health Organization. Global Status Report on Alcohol and Health. Geneva, Switzerland: World Health Organization; 2014.

[6] National Institute on Drug Abuse. Addiction science: from molecules to managed care. Bethesda, MD: National Institute on Drug Abuse; 2008.

[7] Institute of Medicine. Psychosocial Interventions for Mental and Substance Use Disorders: A Framework for Establishing Evidence-based Standards. Washington, DC: National Academies Press; 2015.

[8] Rayan, A. Assessment and Prevention of Risk Factors Associated with Smoking in Jordanian Adolescents: Application of Newman System Model as a Guiding Framework. J Addict Depend. 2016; 2(3): 1-5

[9] Rayan, A., Jaradat, A. Stigma of Mental Illness and Attitudes Toward Psychological Helpseeking in Jordanian University Students. PsycholBehav Sci. 2016; 4 (1).

[10] Rayan, A., Dadoul, A. Decrease the length of hospital stay in depressed cancer patients: Nurses should be involved. Am J Nursing Res. 2015; 3(1) 4-7.
[11] Spiller, H.A., Ryan, M.L., Weston, R.G., et al. Clinical experience with and analytical confirmation of "bath salts" and "legal highs" (synthetic cathinones) in the United States. ClinToxicol (Phila). 2011 49: 499-505.

[12] Weaver, M.F., Schnoll, S.H. In Addictions: A Comprehensive Guidebook. In: McCrady BS, Epstein EE, editors. Stimulants: amphetamines and cocaine. New York: Oxford University Press. 1999: 105-120

[13] Chakraborty, K., Neogi, R., Basu, D. Club drugs: review of the 'rave' with a note of concern for the Indian scenario. Indian J Med Res.2011; 133: 594-604.

[14] Rayan, A., Mahroum, H. The Correlates of Quality Of Life among Jordanian Patients with Major Depressive Disorder. Res PsycholBehav Sci.2016; 4(2): 28-33.

[15] Rayan, A. Assessment and Management of Patients with Drug Abuse: Nurses should be Involved. J Addict Depend. 2017; 3(1): 1- 2.

[16] Foxcroft DR, Tsertsvadze A. Universal schoolbased prevention programs for alcohol misuse in young people. London: Cochrane Database of Systematic Reviews. 2011

[17] Foxcroft DR, Tsertsvadze A. Universal familybased prevention programs for alcohol misuse in young people. London: Cochrane Database of Systematic Reviews. 2011

[18] Siegfried N, Pienaar DC, Ataguba JE, et al. Restricting or banning alcohol advertising to reduce alcohol consumption in adults and adolescents. London: Cochrane Database of Systematic Reviews. 2014

[19] Klimas J, TobinH, Field CA, et al. Psychosocial interventions to reduce alcohol consumption in concurrent problem alcohol and illicit drug users. London: Cochrane Database of Systematic Reviews.2014

[20] Babor T, Higgins-Biddle J. Brief intervention for hazardous and harmful drinking. a manual for use in primary care. Geneva: World Health Organization. 2001

[21] Israel, Y., Hollander, O., Sanchez-Craig, M. \& Booker, S. Screening for problem drinking and counseling by the primary care physician nurse team. Alcoholism: Clinical and Experimental Research. 1996; 20(8), 1443-1450.

[22] Manwell, L.B., et al. Treatment of problem alcohol use in women of childbearing age: Results of a brief intervention trial. Alcoholism: Clinical and Experimental Research. 2000; 24(10), 1517-1524

[23] McManus, S., et al. Implementing an effective intervention for problem drinkers on medical wards.General Hospital Psychiatry. 2003; 25(5), 332-337

[24] Daeppen, J., et al. Brief alcohol intervention and alcohol assessment do not influence 
alcohol use in injured patients treated in the emergency department: A randomized controlled clinical trial. Addiction. 2007; 102(8), 1224-1233

[25] Watson, H.E., Godfrey, C., McFadyen, A., McArthur, K. \& Stevenson, M. Reducing alcohol-related harm in the workplace: a feasibility study of screening and brief interventions for hazardous drinkers. Alcohol Education and Research Council.2009 Available at http://www.aerc.org.uk/ documents/pdfs/finalReports/AERC_FinalRepo rt_0052.pdf, (accessed 28/06/2013).

[26] McIntosh, M.C., Leigh, G., Baldwin, N.J. \&Marmulak, J. Reducing alcohol consumption.Comparing three brief methods in family practice. Canadian Family Physician / Médecin De FamilleCanadien, 1997; 43, 1959

[27] Tomson, Y., Romelsjö, A.\&Aberg, H. Excessive drinking--brief intervention by a primary health care nurse. A randomized controlled trial.Scandinavian Journal of Primary Health Care, 1998;16(3) 188-92.

[28] Ockene, J.K., et al. Brief physician and nurse practitioner-delivered counseling for high-risk drinkers: does it work?.Archives of Internal Medicine, 1999; 159(18), 2198-2205.

[29] Smith, A.J., Hodgson, R.J., Bridgeman, K. \& Shepherd, J.P. A randomized controlled trial of a brief intervention after alcohol-related facial injury. Addiction, 2003; 98(1), 43-52.

[30] Lock, C. A., et al. Effectiveness of nurse-led brief alcohol intervention: a cluster randomized controlled trial. Journal of Advanced Nursing, 2006; 54 (4), 426-439

[31] Holloway, A.S., et al. The effect of brief interventions on alcohol consumption among heavy drinkers in a general hospital setting. Addiction, 2007; 102(11), 1762-1770.

[32] Goodall, C.A., et al. Nurse delivered brief interventions for hazardous drinkers with alcohol-related facial trauma: a prospective randomised controlled trial. The British Journal of Oral and Maxillofacial Surgery, 2008; 46(2), 96-101.

[33] Dent, A.W., Weiland, T.J., Phillips, G.A. \& Lee, N.K. Opportunistic screening and clinician-delivered brief intervention for highrisk alcohol use among emergency department attendees: A randomized controlled trial. Emergency Medicine Australasia, 2008; 20(2), 121-128.

[34] Lane, J., et al. Nurse-provided screening and brief intervention for risky alcohol consumption by sexual health clinic patients. Sexually Transmitted Infections, 2008; 84, 524-527.

[35] Tsai, M., Tsai, Y., Hwang, F. \& Liu, C. Effectiveness of a brief intervention for managing hazardous drinking problems of inpatients in Taiwan. Journal of Advanced Nursing, 2011; 67(9), 2038-2046.

[36] Tsai, Y.F., Tsai, M.C., Lin, Y.P.\&Chen, C.Y. Brief intervention for problem drinkers in a Chinese population: A randomized controlled trial in a hospital setting. Alcoholism: clinical and experimental research, 2009; 33(1):95-101.

[37] Fleming MF, Mundt MP, French MT, Manwell LB, Stauffacher EA \& Barry KL. Brief physician advice for problem drinkers: longterm efficacy and benefit-cost analysis. Alcoholism, Clinical and Experimental Research, 2002; 26(1), 36-43.

[38] Brooker C, Peters J, McCabe C \& Short N. The views of nurses to the conduct of a randomised controlled trial of problem drinkers in an accident and emergency department. International Journal of Nursing Studies, 1999; 36(1), 33-39.

[39] Watson HE A study of minimal interventions for problem drinkers in acute care settings. International Journal of Nursing Studies, 1999; 36(5), 425-434.

[40] Manwell LB, Fleming MF, Mundt MP, Stauffacher EA \& Barry KL Treatment of problem alcohol use in women of childbearing age: Results of a brief intervention trial. Alcoholism: Clinical and Experimental Research, 2000; 24(10), 1517-1524.

[41] Pirskanen M, Laukkanen E \&Pietilä A. A formative evaluation to develop a school health nursing early intervention model for adolescent substance use. Public Health Nursing, 2007; 24(3), 256-256.

[42] Werch CE, Owen DM, Carlson JM, DiClemente CC, Edgemon P \& Moore M. Oneyear follow-up results of the STARS for Families alcohol prevention program. Health Education Research, 2003; 18(1), 74-87.

[43] Watson HE, Godfrey C, McFadyen A, McArthur K \& Stevenson M. Reducing alcohol-related harm in the workplace: a feasibility study of screening and brief interventions for hazardous drinkers. Alcohol Education and Research Council. 2009 http://www.aerc.org.uk/documents/pdfs/final Reports/AERC_ FinalReport_0052.pd f, accessed 25/03/09.

[44] Baker A, Kochan N, Dixon J, Heather N $\&$ Wodak A. Controlled evaluation of a brief intervention for HIV prevention among injecting drug users not in treatment. AIDS Care, 1994; 6(5), 559-570.

[45] Deehan A, Templeton L, Taylor C, Drummond C \&Strang J. Are practice nurses an unexplored resource in the identification and management of alcohol misuse? Results from a study of practice nurses in England and Wales in 1995. Journal of Advanced Nursing, 1998; 28(3), 592-597. 
[46] Owens L, Gilmore IT \&Pirmohamed M. General practice nurses' knowledge of alcohol use and misuse: a questionnaire survey. Alcohol and Alcoholism, 2000; 35(3), 259-262.

[47] Lappalainen-Lehto R, Seppä K \&NordbackI Cutting down substance abuse - present state and visions among surgeons and nurses. Addictive Behaviors, 2007; 30(5), 1013-1018.

[48] Kerker BD, Horwitz SM \& Leventhal JM. Patients' characteristics and providers' attitudes: Predictors of screening pregnant women for illicit substance use. Child Abuse and Neglect,2004; 28(2), 209-209.

[49] AndreassonS, Hjalmarsson K \&Rehnman C. Implementation and dissemination of methods for prevention of alcohol problems in primary health care: a feasibility study. Alcohol and Alcoholism, 2000; 35(5), 525-530.

[50] Chang JC, Dado D, Frankel RM, Rodriguez KL, Zickmund S, Ling BS et al. When pregnant patients disclose substance use: Missed opportunities for behavioral change counseling. Patient Education and Counseling, 2008; 72(3), 394-401.

[51] Davis P \&Carr TL. Needs assessment and current practice of alcohol risk assessment of pregnant women and women of childbearing age by primary health care professionals. Canadian Journal of Clinical Pharmacology, 2008; 15(2), 214-222.

[52] Herzig K, Danley D, Jackson R, Petersen R, Chamberlain L \&Gerbert B. Seizing the 9month moment: Addressing behavioural risks in prenatal patients. Patient Education and Counseling, 2006; 61, 228-235.

[53] Ozer EM, Adams SH, Gardner LR, Mailloux DE, Wibbelsman CJ \& Irwin Jr CE. Provider self-efficacy and the screening of adolescents for risky health behaviors. Journal of Adolescent Health, 2004; 35(2), 101-107.

[54] Griffiths RD, Stone A, Tran DT, Fernandez RS \& Ford K. Drink a little; take a few drugs: do nurses have knowledge to identify and manage in-patients at risk of drugs and alcohol? Drug and Alcohol Review, 2007; 26(5), 545-552.

[55] Bayo L et al. Effectiveness of a nurse-led cognitive behavioural therapy intervention in the management of substance abuse amongst selected secondary school students in Ejigbo local government area, Osun state, Nigeria. Int J Med Res Rev 2015; 3(11):1345-1352. doi: 10.17511/ijmrr.2015.i11.244.

[56] Austin, W., \& Boyd, M. Psychiatric and mental health nursing for Canadian practice (2nd ed.). Philadelphia, PA: Lippincott Williams \& Wilkins. 2010.

[57] Simpson, P. Family assessment and interventions. In W. Austin \& M. A. Boyd (Eds.), Psychiatric and mental health nursing for Canadian practice (2nd ed.) (pp. 291-307). Philadelphia, PA: Lippincott Williams \& Wilkins. 2010.

[58] Substance Abuse and Mental Health Services Administration (SAMHSA). Family behavior therapy. (2014a). Retrieved from http://www.nrepp.samhsa.gov/ViewInterventio n.aspx?id=113

[59] Nyamathi A, Branson C, Kennedy B, Salem B, Khalilifard F, Marfisee M, Getzoff D, Leake B. Impact of nursing intervention on decreasing substances among homeless youth. The American journal on addictions. 2012 Nov; 21(6):558-65.

Citation: Jaison Joseph, Dr.Deepika C Khakha, Biji P Varkey, Nurse-Led Interventions in the De-Addiction Setting: Current State of Evidence. ARC Journal of Nursing and Healthcare. 2020; 6(1):10-15. DOI: doi.org/ 10.20431/2455-4324.0601002.

Copyright: () 2020 Authors. This is an open-access article distributed under the terms of the Creative Commons Attribution License, which permits unrestricted use, distribution, and reproduction in any medium, provided the original author and source are credited. 